\title{
Cerebral cross-perfusion and the circle of Willis: does physiology trump anatomy?
}

This article was published in the following Dove Press journal:

Journal of Vascular Diagnostics and Interventions

20 July 2017

Number of times this article has been viewed

\author{
Korana Musicki' \\ Katherine Victoria Hurst ${ }^{1,2}$ \\ Zoltán Molnár ${ }^{3}$ \\ Elizabeth Hardy ${ }^{4}$ \\ Ashok Handa ${ }^{1,2}$ \\ 'Department of Vascular Surgery, \\ John Radcliffe Hospital, ${ }^{2}$ Nuffield \\ Department of Surgical Sciences, \\ ${ }^{3}$ Department of Physiology, Anatomy \\ and Genetics, University of Oxford, \\ ${ }^{4}$ Vascular Studies Unit, John Radcliffe \\ Hospital, Oxford, UK
}

Introduction: Cerebral cross-perfusion is essential for ipsilateral brain viability during unilateral insult. Aortic arch and great vessel procedures depend on its function for safe practice, unless adjuncts like shunts are used. This paper assesses the contribution of cerebrovascular anatomy against physiology in determining requisite hemispheric perfusion during carotid endarterectomy (CEA).

Materials and methods: A review of shunting requirements for CEAs under locoregional anesthesia (LA) at the John Radcliffe Hospital during 1999-2013 was performed. A PubMed search for "Circle of Willis" was screened for all original articles defining cerebrovascular anatomy by postmortem or angiography.

Results: Over 14 years, 1137 CEAs were performed under cervical plexus block; during this period, the departmental standard of practice evolved from exclusively general anesthesia to predominantly LA. CEAs performed under LA during the early phase (1999-2003) had a shunt rate of $15.1 \%$, compared to $20 \%$ as predicted by stump pressures alone. However, shunting decreased as higher perioperative systolic pressures were routinely practiced; shunt rates were $8.0 \%$ during the intermediate (2004-2007) and 6.4\% during the later (2008-2013) phase. By comparison, 25 articles characterizing 6414 brains report an intact circle of Willis in 33-35\% of people, with a complete hemi-circle anteriorly $(77 \%)$ seen more commonly than posteriorly (42\%), and $11-16 \%$ deficient in both hemi-circles with no cross-flow.

Conclusion: Cerebral cross-perfusion is fundamental for safe CEA. Anatomy of the circle of Willis alone does not itself determine adequacy. Physiological mechanisms are important in overriding apparent deficiencies, and these can be manipulated perioperatively. Consequently, only 1 out of 15 patients requires shunting during CEA.

Keywords: Circle of Willis, cerebral, cross-perfusion, carotid, shunt, anatomical variation

\section{Introduction}

Cerebral function is of utmost importance to survival, and unsurprisingly, there are multiple overlapping anatomical and physiological mechanisms by which adequate cerebral perfusion is maintained. Cerebral vascular anatomy consequently features many large- and small-scale, seemingly redundant, arterial networks to allow crossperfusion should any single major vessel occlude, including the well-characterized circle of Willis (Figure 1). ${ }^{1}$ However, various anatomical studies, including postmortem dissections and imaging series, indicate that the circle of Willis manifests in complete configuration in less than half the population. ${ }^{2,3}$

Physiological mechanisms appear to have a significant role in maintaining cerebral perfusion, not only by phenomena such as cerebral autoregulation, which allows for
Correspondence: Ashok Handa Nuffield Department of Surgical Sciences, John Radcliffe Hospital, Oxford OX3 9DU, UK

Tel +44 I865 $22 \quad 1278$

Fax +44186522 III

Email Ashok.Handa@nds.ox.ac.uk 


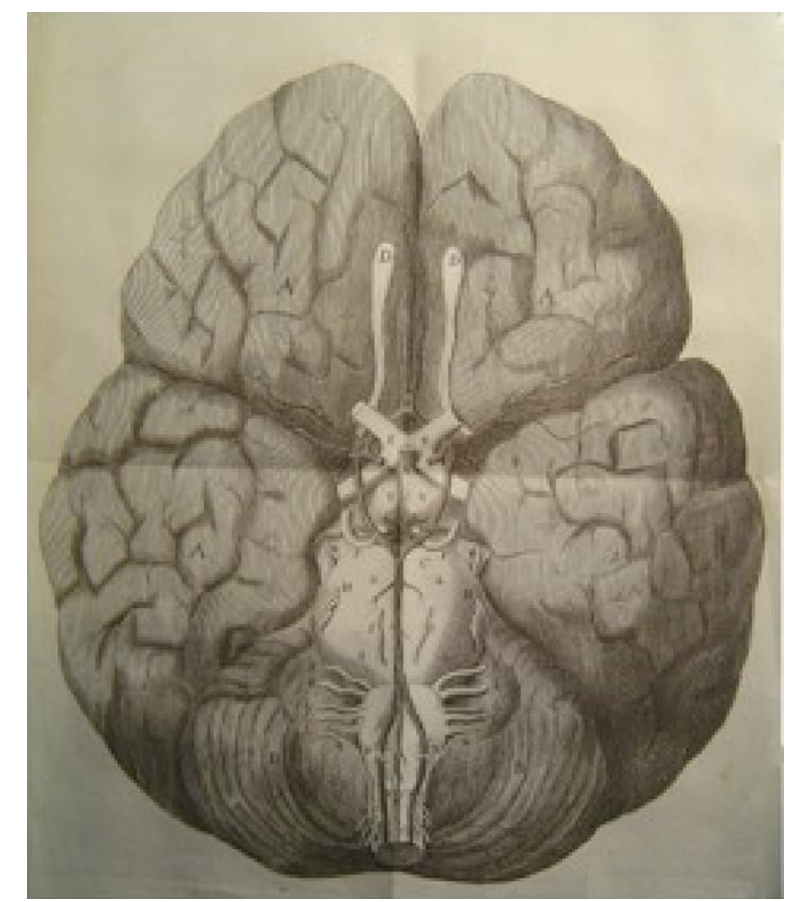

Figure I Original illustration of the cerebral arterial circle by $\mathrm{Dr}$ Thomas Willis (1664).

Note: Reproduced with the permission of the library of St John's College, Oxford.

constant flow over a broad range of systemic mean arterial pressures, but also cerebral cross-perfusion. ${ }^{4}$ Logically, the fate of the ipsilateral cerebral cortex during unilateral vascular deficiency depends on the ability of the individual to activate these cerebral cross-perfusion pathways.

Aortic arch and great vessel interventions depend on cerebral anatomical and physiological homeostatic mechanisms for safety, unless adjuncts like shunts can be used. However, preoperative imaging and perioperative monitoring are notoriously poor predictors of patient response during intraoperative manipulation, including carotid cross-clamping during carotid surgery or selective antegrade cerebral perfusion during aortic arch surgery.,

This study examines the relative contribution of cerebrovascular anatomy and physiology in maintaining adequate hemispheric perfusion, specifically by quantifying the population at risk of poor cerebral cross-perfusion due to anatomical circle of Willis deficiencies, and comparing it to the population that required shunting during carotid cross-clamping.

A secondary outcome of the study is to assess overall shunt rates during carotid endarterectomy (CEA), and in light of this, any consequent implications on surgical training.

\section{Materials and methods}

\section{Anatomical data}

A PubMed search using the keyword "Circle of Willis" was performed. All articles were screened for original research pieces quantifying human variations of the circle of Willis. For each original paper, mode of assessment, ethnicity of the population and proportions of circle of Willis variants were recorded; specifically, patient populations were stratified according to completeness and deficiencies in both the anterior and posterior hemi-circles.

\section{Physiological data}

An audit of consecutive CEAs performed under locoregional anesthesia (LA) at the John Radcliffe Hospital during 19992013 was compiled. Superficial cervical plexus block was applied, and hemodynamic monitoring was by radial arterial line. Carotid bifurcation exposure and endarterectomy were performed by techniques of choice of the consultant vascular surgeon; these included eversion or standard endarterectomy, with or without vein or prosthetic patch anastomotic repair. Unilateral carotid occlusion tolerance was monitored by regular gross neurological assessment, which included patients' recall of their own full name, date of birth and residential address, in addition to contralateral handgrip and ankle plantar flexion power, as well as general agitation. The necessity for shunting was determined perioperatively at the discretion of the consultant vascular surgeon, and typically occurred if clamp intolerance manifested early during the routine 5-min internal carotid artery (ICA) test clamp (prior to arteriotomy), or during the endarterectomy or anastomotic repair.

Over the 14-year period described, there were 3 distinct eras in surgical development. Era 1 (1999-2003) denotes the learning curve for surgeons and anesthetists following the introduction of local anesthetic. Shunts were used for all patients who had neurological defect during test clamping. In Era 2 (2004-2007), any neurological deficit during test clamping was initially dealt with by raising the systolic pressure and repeating a trial clamp. Only if a residual neurological deficit was present would shunting proceed. In Era 3 (2008-2013), any neurological deficit during trial clamping was responded to with both a raise of systolic pressure and increased arterial oxygenation.

\section{Results}

\section{Anatomical data}

The PubMed search utilizing the keyword "Circle of Willis" yielded 2675 publically available articles; however, only 34 papers described a distinct human population case series that could be assessed. The total publications finally analyzed were reduced to 25 that contained comparable numerical anatomical descriptions, which together reported a total of 6414 individual cerebrovascular anatomies of diverse ethnic backgrounds. 
Of 14 postmortem dissection reports, assessing 4393 individual brains, a complete circle of Willis was seen in $35 \%$ of cadaveric specimens. It was noted that the complete anterior portion was more common (77\%) than a complete posterior portion (42\%) (Table 1). Of 11 angiographic studies (computed tomography, magnetic resonance or digital subtraction modalities) examining 2021 vasculatures, a complete circle of Willis was seen in 33\% of all scans. Again, a complete anterior portion was observed more commonly (77\%) than a complete posterior portion (42\%) (Table 2). Overall, the anatomical data indicated that $11 \%$ (radiological) to $16 \%$ (cadaveric) of the population had deficiencies in both anterior and posterior hemi-circles.

\section{Physiological data}

The John Radcliffe Hospital Department of Vascular Surgery evolved its CEA practice from exclusively general anesthesia (GA) to predominantly LA circa 1999 . Over the 14-year study period since LA uptake, 1137 CEAs were performed under cervical plexus block (Table 3). Initial rates of shunting in our unit showed a mean of $15.1 \%$ during 1999-2003, following a similar trend to the anatomy data. However, during 2004-2007, a shunt rate of $8.0 \%$ was observed, and this further decreased to $6.4 \%$ during 2008-2013.

International experience with CEA under LA demonstrates a reported average shunt rate of $15 \%$; however, only a Korean group has managed to reduce it to $4 \%$ (Table 4 ). ${ }^{7}$

\section{Discussion}

Cerebral cross-perfusion is essential in preserving ipsilateral brain survival and function during unilateral vascular interruption. Various mechanisms have been described including anatomical collaterals $\mathrm{s}^{8-11}$ and physiological alterations, ${ }^{4}$ but neither preoperative scanning nor perioperative surrogate physiological cerebral monitoring has been a good predictor of postoperative outcomes.

Anatomical explanations for cross-perfusion have relied on a series of collateral pathways that are deemed essential. First described by Dr Thomas Willis in 1664, the eponymous intracranial circle, loop or polygon as it is variously referred to, has been the benchmark respected by generations of physicians and anatomists. However, despite the perceived importance of this intracranial network, multiple human variations exist, including hypoplastic and aplastic communicating vessels that frequently result in incomplete hemicircles, with no apparent disadvantages. Perhaps due to the "higher" end organ significance or due to the less individual elements, the anterior circulation tends to be complete in a greater proportion of the population than the posterior circulation. ${ }^{12-14}$ Interestingly, $11-16 \%$ of the population have no evidence of classic anatomical pathways allowing for cross-brain perfusion. ${ }^{1,13,15}$

Alternative intracranial pathways include the leptomeningeal anastomoses of Heubner and micronetworks of Schmidt and Pfeiffer, which collateralize the ipsilateral anterior circulation only. ${ }^{16}$ Supplementary extracranial collateral pathways that can develop include external to internal carotid anastomoses, via the superficial temporal and middle maxillary arteries, to supraorbital and dorsal nasal branches of the ophthalmic artery, respectively. These certainly become important sources of alternate blood flow if hemodynamically significant lesions develop at the ICA origin, as typically

Table I Anatomical data based on postmortem dissections across various mono- and multiethnic groups

\begin{tabular}{|c|c|c|c|c|c|c|c|}
\hline Report & Year & Ethnicity & Total & Complete (\%) & Anterior (\%) & Posterior (\%) & Deficient (\%) \\
\hline Papantchev et a ${ }^{22}$ & 2013 & Bulgarian & 250 & 41 & 86 & 49 & 6 \\
\hline lqbal'2 & 2013 & Indian & 50 & 60 & 92 & 68 & 0 \\
\hline Hashemi et al ${ }^{13}$ & 2013 & Iranian & 200 & 46 & 82 & 53 & 12 \\
\hline Siddiqi et $\mathrm{al}^{23}$ & 2013 & Pakistani & 51 & 41 & 84 & 41 & 16 \\
\hline De Silva et $\mathrm{a}^{24}$ & 2011 & Sri Lankan & 220 & 15 & 52 & 32 & 31 \\
\hline Manninen et $\mathrm{a}^{25}$ & 2009 & Finnish & 92 & 21 & 76 & 39 & 5 \\
\hline Kapoor et al" & 2008 & Indian & 1000 & 66 & 94 & 72 & 8 \\
\hline Ardakani et al ${ }^{14}$ & 2008 & Iranian & 30 & 43 & 100 & 43 & 0 \\
\hline Eftekhar et al ${ }^{15}$ & 2006 & Iranian & 92 & 32 & 88 & 33 & 12 \\
\hline el Khamlichi et al ${ }^{9}$ & 1985 & Moroccan & 100 & 18 & 60 & 31 & 26 \\
\hline Lazorthes and Gouazé26 & 1979 & French & 200 & 15 & 58 & 21 & 37 \\
\hline Fisher et $\mathrm{al}^{27}$ & 1965 & USA & 414 & 5 & 58 & 6 & 38 \\
\hline Riggs and Rupp ${ }^{28}$ & 1963 & USA & 994 & 19 & 52 & 32 & 35 \\
\hline Fawcett and Blachford ${ }^{10}$ & 1905 & UK & 700 & 73 & 100 & 73 & 0 \\
\hline Total & 14 & & 4393 & & & & \\
\hline Mean & & & & 35 & 77 & 42 & 16 \\
\hline
\end{tabular}

Notes: The circle of Willis configurations were designated as "complete" (if no segments were aplastic or hypoplastic), "anterior" (if no segments were aplastic or hypoplastic in the anterior portion only), "posterior" (if no segments were aplastic or hypoplastic in the posterior portion only) or "deficient" (if segments were aplastic or hypoplastic in both). 
Table 2 Anatomical data based on radiological studies across various mono- and multiethnic groups

\begin{tabular}{|c|c|c|c|c|c|c|c|c|}
\hline Report & Year & Ethnicity & Total & Complete (\%) & Anterior (\%) & Posterior (\%) & Deficient (\%) & Mode \\
\hline Papantchev et a ${ }^{22}$ & 2013 & Bulgarian & 250 & 41 & 86 & 49 & 6 & CTA \\
\hline Pennekamp et $\mathrm{a}^{21}$ & 2013 & Dutch & 140 & 9 & 80 & 15 & 14 & CTA \\
\hline Pennekamp et $\mathrm{al}^{21}$ & 2013 & Dutch & 284 & 9 & 83 & 32 & 0 & MRA \\
\hline Cho et $\mathrm{al}^{7}$ & 2013 & Korean & 45 & 29 & 60 & 60 & 9 & CTA \\
\hline $\begin{array}{l}\text { Klimek-Piotrowska } \\
\text { et a }{ }^{29}\end{array}$ & 2013 & Polish & 250 & 17 & 47 & 27 & 43 & CTA \\
\hline Chuang et $\mathrm{al}^{30}$ & 2013 & Taiwanese & 64 & 33 & 81 & 52 & 0 & CTA \\
\hline $\mathrm{Li}$ et $\mathrm{al}^{31}$ & 2011 & Chinese & 160 & 27 & 79 & 31 & 17 & CTA \\
\hline Cavestro et $\mathrm{al}^{8}$ & 2011 & Italian & 429 & 67 & 88 & 75 & 4 & MRA \\
\hline Urbanski et al ${ }^{32}$ & 2008 & German & 99 & 60 & $\mathrm{~N} / \mathrm{A}$ & N/A & 9 & CTA \\
\hline Waaijer et al ${ }^{33}$ & 2007 & Dutch & 182 & 24 & 80 & 29 & 15 & CTA \\
\hline Macchi et $\mathrm{al}^{34}$ & 2002 & Italian & 118 & 47 & 90 & 48 & 8 & MRA \\
\hline Total & 11 & & 2021 & & & & & \\
\hline Mean & & & & 33 & 77 & 42 & 11 & \\
\hline
\end{tabular}

Notes: These include CTA, MRA and digital subtraction angiography. The circle of Willis configurations were designated as "complete" (if no segments were aplastic or hypoplastic), "anterior" (if no segments were aplastic or hypoplastic in the anterior portion only), "posterior" (if no segments were aplastic or hypoplastic in the posterior portion only) or "deficient" (if segments were aplastic or hypoplastic in both).

Abbreviations: CTA, computed tomography angiography; MRA, magnetic resonance angiography; N/A, not available.

Table 3 Physiological data based on the John Radcliffe Hospital experience of carotid endarterectomy under locoregional anesthesia over specific time periods

\begin{tabular}{lllll}
\hline Year & Ethnicity & Total (n) & Shunt (n) & Shunt (\%) \\
\hline 1999-2003 & Mixed UK residents & 199 & 30 & 15.1 \\
2004-2007 & Mixed UK residents & 363 & 29 & 8.0 \\
2008-2013 & Mixed UK residents & 575 & 37 & 6.4 \\
Total & & 1137 & & \\
\hline
\end{tabular}

Table 4 Physiological data based on the perioperative experience of carotid endarterectomy under locoregional anesthesia across various vascular surgical units and ethnic groups

\begin{tabular}{|c|c|c|c|c|c|}
\hline Report & Year & Ethnicity & Total (n) & Shunt (n) & Shunt (\%) \\
\hline Pennekamp et $\mathrm{al}^{21}$ & 2013 & Dutch & 431 & 65 & 15 \\
\hline Cho et $\mathrm{al}^{7}$ & 2013 & Korean & 45 & 2 & 4 \\
\hline Lee et $\mathrm{al}^{20}$ & 2004 & Korean & 117 & 23 & 20 \\
\hline Kim et al ${ }^{19}$ & 2002 & Korean & 67 & 14 & 21 \\
\hline Total & 4 & & 660 & & \\
\hline Mean & & & & 26 & 15 \\
\hline
\end{tabular}

occurs due to atherosclerotic disease, although they may not be able to compensate during acute occlusion.

Perioperative proxy cerebral physiological monitoring during GA has been notoriously hampered by interpretation inaccuracies. Stump pressure assessment is performed following clamping the common carotid and external carotid arteries by needling the ICA connected to an arterial transducer; arbitrarily, pressures above $50 \mathrm{mmHg}$ are deemed to be adequate. Using this technique, shunt rates are around $20 \%$; however, false positives are $1.45 \%$, and negatives are $70.4 \%{ }^{5}$ Transcranial Doppler is performed by setting the probe to monitor the flow in the ipsilateral middle cerebral artery and monitoring for changes in peak systolic velocity. Using this technique, shunt rates are $11 \%$; however, false positives are $2 \%$, and negatives are $29 \%{ }^{6}$ Electroencephalography with somatosensory evoked potential monitoring requires up to 16 leads to monitor changes in background brain electrical activity. Using this technique, shunt rates are around $6 \%$; however, false positives are approximately $1 \%$, and negatives are up to $40.6 \%{ }^{5}$ Due to the complexities and inaccuracies of these techniques, when performing CEA under GA, the surgical community is divided into the majority routine-shunters and minority selective-shunters, the latter of which may use any of the above techniques with a clinical assessment of strong pulsatile backbleeding.

The gold standard for cerebral hemispheric tolerance of unilateral carotid occlusion is a neurological assessment. ${ }^{17}$ This can easily be performed if the procedure is done under LA and with the application of a hemostatic clamp or an occlusive balloon at the ICA origin. Certainly, the initial John Radcliffe Hospital experience with shunt rates of $15.1 \%$ on average seemed to appropriately reflect the anatomical data of doubly deficient circle of Willis variants at $11-16 \%$. However, with physiological manipulation, the shunt rates have progressively decreased to $6.4 \%$ despite the population group remaining the same. This is mostly attributed to the deliberate increase in mean arterial pressures during cross-clamping; many patients will reflexively increase their own systemic blood pressure through the process of autoregulation; however, some require additional support, typically with addition of boluses of metaraminol.

The latest Vascular Society Quality Improvement Program (VSQIP) report 2015 shows that the John Radcliffe Hospital has performed $260 \mathrm{CEAs}$ over a 3 -year period, with an overall 
stroke and death rate of $2.1 \%$, despite a $6.4 \%$ shunt rate. This is well within the top decile of outcomes in the UK. ${ }^{18}$

The experience and physiological data, with respect to CEA under LA, have also been published by other units with various results. The Korean experience appears to be similar, with initial publications reporting shunt rates of $21 \%$ in 67 patients ${ }^{19}$ and $20 \%$ in 117 patients, ${ }^{20}$ while more contemporary articles report shunt rates as low as $4 \%$ in 45 patients. ${ }^{7}$ However, the Dutch experience remains more conservative, with shunt rates remaining at $15 \%$ in 431 patients. $^{21}$

Cerebral cross-perfusion is fundamental for safe completion of CEA. Anatomy of the circle of Willis alone does not itself determine adequacy. Physiological mechanisms are important in overriding apparent deficiencies, and these can be manipulated perioperatively. With such measures, only 1 in every 15 patients may require shunting during CEA, and this could have implications for the training of future vascular surgeons.

\section{Acknowledgments}

The authors would like to thank the vascular sonographers K Bond, R Davies and A Lehmann, and the vascular surgeons J Collin, C Darby, A Halliday, L Hands, T Magee, J Perkins, P Rutter and E Sideso for their assistance.

\section{Disclosure}

The authors report no conflicts of interest in this work.

\section{References}

1. Vrselja Z, Brkic H, Mrdenovic S, Radic R, Curic G. Function of circle of Willis. J Cereb Blood Flow Metab. 2014;34(4):578-584.

2. Klimek-Piotrowska W, Rybicka M, Wojnarska A, Wójtowicz A, Koziej M, Hołda MK. A multitude of variations in the configuration of the circle of Willis: an autopsy study. Anat Sci Int. 2016;91(4):325-333.

3. Macchi C, Catini C, Federico C, et al. Magnetic resonance angiographic evaluation of circulus arteriosus cerebri (circle of Willis): a morphologic study in 100 human healthy subjects. Ital J Anat Embryol. 1996;101(2):115-123.

4. Stoneham MD, Thompson JP. Arterial pressure management and carotid endarterectomy. Br J Anaesth. 2009;102(4):442-452.

5. Hans SS, Jareunpoon O. Prospective evaluation of electroencephalography, carotid artery stump pressure, and neurologic changes during 314 consecutive carotid endarterectomies performed in awake patients. J Vasc Surg. 2007;45(3):511-515.

6. Cao P, Giordano G, Zannetti S, et al. Transcranial Doppler monitoring during carotid endarterectomy: is it appropriate for selecting patients in need of a shunt? JVasc Surg. 1997;26(6):973-979; discussion 979-980.

7. Cho J, Lee KK, Yun WS, Kim HK, Hwang YH, Huh S. Selective shunt during carotid endarterectomy using routine awake test with respect to a lower shunt rate. J Korean Surg Soc. 2013;84(4):238-244.

8. Cavestro C, Richetta L, L'episcopo MR, Pedemonte E, Duca S, Di Pietrantonj C. Anatomical variants of the circle of Willis and brain lesions in migraineurs. Can J Neurol Sci. 2011;38(3):494-499.

9. el Khamlichi A, Azouzi M, Bellakhdar F, et al. Configuration anatomique du polygone de Willis de l'adulte, étudié par les techniques d'injection. A propos de 100 cerveaux. [Anatomic configuration of the circle of Willis in the adult studied by injection technics. Apropos of 100 brains]. Neurochirurgie. 1985;31(4):287-293. French [with English abstract].
10. Fawcett E, Blachford JV. The Circle of Willis: an examination of 700 specimens. J Anat Physiol. 1905;40(Pt 1):63.2-70.

11. Kapoor K, Singh B, Dewan LI. Variations in the configuration of the circle of Willis. Anat Sci Int. 2008;83(2):96-106.

12. Iqbal S. A comprehensive study of the anatomical variations of the circle of Willis in adult human brains. J Clin Diagn Res. 2013;7(11):2423-2427.

13. Hashemi SM, Mahmoodi R, Amirjamshidi A. Variations in the anatomy of the Willis' circle: a 3-year cross-sectional study from Iran (20062009). Are the distributions of variations of circle of Willis different in different populations? Result of an anatomical study and review of literature. Surg Neurol Int. 2013;4:65.

14. Ardakani SK, Dadmehr M, Nejat F, et al. The cerebral arterial circle (circulus arteriosus cerebri): an anatomical study in fetus and infant samples. Pediatr Neurosurg. 2008;44(5):388-392.

15. Eftekhar B, Dadmehr M, Ansari S, Ghodsi M, Nazparvar B, Ketabchi E. Are the distributions of variations of circle of Willis different in different populations? - results of an anatomical study and review of literature. BMC Neurol. 2006;6:22.

16. Gorczyca W, Mohr G. Microvascular anatomy of Heubner's recurrent artery. Neurol Res. 1987;9(4):259-264.

17. Silbert BS, Koumoundouros E, Davies MJ, Cronin KD. Comparison of the processed electroencephalogram and awake neurological assessment during carotid endarterectomy. Anaesth Intensive Care. 1989;17(3): 298-304.

18. Vascular Society. Reports. Available from: https://www.vsqip.org.uk/ reports/. Accessed March, 2017.

19. Kim GE, Cho YP, Lim SM. The anatomy of the circle of Willis as a predictive factor for intra-operative cerebral ischemia (shunt need) during carotid endarterectomy. Neurol Res. 2002;24(3):237-240.

20. Lee JH, Choi CG, Kim DK, Kim GE, Lee HK, Suh DC. Relationship between circle of Willis morphology on 3D time-of-flight MR angiograms and transient ischemia during vascular clamping of the internal carotid artery during carotid endarterectomy. AJNR Am J Neuroradiol. 2004;25(4):558-564.

21. Pennekamp CW, van Laar PJ, Hendrikse J, et al. Incompleteness of the circle of Willis is related to EEG-based shunting during carotid endarterectomy. Eur J Vasc Endovasc Surg. 2013;46(6):631-637.

22. Papantchev V, Stoinova V, Aleksandrov A, et al. The role of Willis circle variations during unilateral selective cerebral perfusion: a study of 500 circles. Eur J Cardiothorac Surg. 2013;44(4):743-753.

23. Siddiqi H, Tahir M, Lone KP. Variations in cerebral arterial circle of Willis in adult Pakistani population. J Coll Physicians Surg Pak. 2013; 23(9):615-619.

24. De Silva KR, Silva R, Gunasekera WS, Jayesekera RW. Prevalence of typical circle of Willis and the variation in the anterior communicating artery: a study of a Sri Lankan population. Ann Indian Acad Neurol. 2009; 12(3):157-161.

25. Manninen H, Mäkinen K, Vanninen R, Ronkainen A, Tulla H. How often does an incomplete circle of Willis predispose to cerebral ischemia during closure of carotid artery? Postmortem and clinical imaging studies. Acta Neurochir (Wien). 2009;151(9):1099-1105.

26. Lazorthes G, Gouazé A. Conception fonctionnelle du polygone de Willis [Functional concept of the circle of Willis]. C R Assoc Anat. 1970;149:826-836. French [with English abstract].

27. Fisher ER, Davis JS, Lemmen LJ. Anomalous reduplication of the circle of Willis. J Neurosurg. 1959;16(3):331-336.

28. Riggs HE, Rupp C. Variation in form of circle of Willis. The relation of the variations to collateral circulation: anatomic analysis. Arch Neurol. $1963 ; 8: 8-14$.

29. Klimek-Piotrowska W, Kopeć M, Kochana M, et al. Configurations of the circle of Willis: a computed tomography angiography based study on a Polish population. Folia Morphol (Warsz). 2013;72(4): 293-299.

30. Chuang YM, Chan L, Lai YJ, et al. Configuration of the circle of Willis is associated with less symptomatic intracerebral hemorrhage in ischemic stroke patients treated with intravenous thrombolysis. JCrit Care. 2013;28(2):166-172. 
31. Li Q, Li J, Lv F, Li K, Luo T, Xie P. A multidetector CT angiography study of variations in the circle of Willis in a Chinese population. J Clin Neurosci. 2011;18(3):379-383.

32. Urbanski PP, Lenos A, Blume JC, et al. Does anatomical completeness of the circle of Willis correlate with sufficient cross-perfusion during unilateral cerebral perfusion? Eur J Cardiothorac Surg. 2008;33(3):402-408.
33. Waaijer A, van Leeuwen MS, van der Worp HB, Verhagen HJ, Mali WP, Velthuis BK. Anatomic variations in the circle of Willis in patients with symptomatic carotid artery stenosis assessed with multidetector row CT angiography. Cerebrovasc Dis. 2007;23(4):267-274.

34. Macchi C, Lova RM, Miniati B, et al. The circle of Willis in healthy older persons. J Cardiovasc Surg (Torino). 2002;43(6):887-890.

\section{Publish your work in this journal}

The Journal of Vascular Diagnostics and Interventions is an international, peer-reviewed journal of diagnostics, focusing on non invasive vascular investigation methods involved in the evaluation of vascular diseases. The journal is committed to the rapid publication in the fields of vascular diseases. Original research, review, case reports, expert opinion and commentaries are all considered for publication. The manuscript management system is completely online and includes a very quick and fair peer-review system, which is all easy to use. Visit http://www.dovepress.com/testimonials.php to read real quotes from published authors. 